\title{
Propriedades Físicas da Madeira de Teca Termorretificada
}

\author{
Juliana de Oliveira Lopes ${ }^{1}$, Rosilei Aparecida Garcia ${ }^{2}$, \\ Alexandre Miguel do Nascimento ${ }^{2}$, João Vicente de Figueiredo Latorraca ${ }^{2}$ \\ ${ }^{1}$ Programa de Pós-Graduação em Ciências Ambientais e Florestais, Universidade Federal Rural do Rio de Janeiro - UFRRJ, \\ Seropédica/RJ, Brasil \\ ${ }^{2}$ Departamento de Produtos Florestais - DPF, Instituto de Florestas - IF, Universidade Federal Rural do Rio de Janeiro - UFRRJ, \\ Seropédica/RJ, Brasil
}

\section{RESUMO}

O objetivo deste estudo foi determinar o efeito de tratamentos termorretificadores nas propriedades físicas (densidade e teor de umidade) da madeira de teca (Tectona grandis L. f.), proveniente de plantios de diferentes espaçamentos. Amostras de madeira de cerne e alburno foram termorretificadas a $180^{\circ} \mathrm{C}$ e $200^{\circ} \mathrm{C}$ durante 2 horas e 30 minutos. A termorretificação afetou mais a densidade do cerne do que a densidade do alburno. As madeiras de cerne e alburno apresentaram uma redução média de $52 \%$ no teor de umidade de equilíbrio, após a termorretificação. O espaçamento entre árvores também afetou as propriedades físicas da madeira após a termorretificação.

Palavras-chave: densidade, teor de umidade, termorretificação, Tectona grandis.

\section{Physical Properties of Heat-treated Teak Wood}

\begin{abstract}
The objective of this study was to determine the effect of heat treatments on the physical properties (density and moisture content) of teak (Tectona grandis L. f.) wood from plantations of different spacings. Heartwood and sapwood samples were heat treated at $180{ }^{\circ} \mathrm{C}$ and $200{ }^{\circ} \mathrm{C}$ for 2 hours and 30 minutes. Heat treatment produced greater effect on heartwood density compared with sapwood density. Heartwood and sapwood presented an average reduction of $52 \%$ in equilibrium moisture content after heat treatment. Tree spacing also affected the physical properties of wood after heat treatment.
\end{abstract}

Keywords: density, moisture content, heat treatment, Tectona grandis. 


\section{INTRODUÇÃO}

A termorretificação é um tratamento que permite melhorar a estabilidade dimensional e a durabilidade natural da madeira, e que vem ganhando destaque ultimamente por ser um tratamento preservativo ecologicamente amigável, visto que é livre de produtos químicos nocivos ao meio ambiente e, pela madeira tratada ser 100\% reciclável, sem riscos de contaminação de solos (Viitaniemi \& Jämsä, 1994; Kamdem \& Pizzi, 2002; Esteves et al., 2007; Garcia et al., 2012). Atualmente, a madeira termorretificada vem sendo comercializada, principalmente na Europa, onde vários processos foram patenteados, os quais são conhecidos como Retification ${ }^{\circledR}$ e Perdure ${ }^{\circledR}$ (França); Oil Heat Treatment-OHT (Alemanha); Plato ${ }^{\circledR}$ Wood «Proving Lasting Advanced Timber Option» (Países Baixos) e Thermowood ${ }^{\circledR}$ (Finlândia) ou Thermoholz ${ }^{\circledR}$ (Áustria) (Rapp, 2001; Militz, 2002; Oltean et al., 2007). As principais diferenças entre estes processos são a utilização de oxigênio ou nitrogênio, a presença ou ausência de vapor, o processo seco ou úmido, e a utilização de óleos (Militz, 2002).

Syrjänen (2001) afirma que a propriedade mais importante da madeira termorretificada é o menor teor de umidade de equilíbrio higroscópico, o que, consequentemente, reduz as variações dimensionais (contração e inchamento) do material, quando comparada à madeira não tratada.

Weiland \& Guyonnet (2003) e Rousset et al. (2004) afirmam que a madeira termorretificada apresenta uma maior estabilidade dimensional devido à redução da higroscopicidade, causada pela degradação das hemiceluloses (constituinte mais higroscópico da parede celular), à ruptura dos grupos hidroxílicos livres da região amorfa da celulose e à reticulação dos polímeros que compõem a madeira.

Além de maior estabilidade dimensional e maior durabilidade, a madeira apresenta uma alteração na sua cor original após a termorretificação. Estudos realizados por Lopes (2012) mostraram que a termorretificação pode promover uma uniformidade na cor da madeira de teca (Tectona grandis L. f.), valorizando assim o produto final. Segundo Möttönen et al. (2002), a uniformidade da cor da madeira é um atributo estético importante e apresenta um impacto direto no estabelecimento do preço final do produto.

Apesar das vantagens estéticas, a madeira termorretificada apresenta perda de massa, a qual afeta a sua densidade e, consequentemente, as suas propriedades mecânicas. Vários trabalhos mostram que ocorre uma perda de massa progressiva, com o aumento do tempo e da temperatura de aquecimento (Hakkou et al., 2005; Garcia et al., 2012). Entretanto, a perda de massa e a redução da densidade e da resistência mecânica da madeira também dependem da espécie e do teor de umidade. De acordo com Kamdem \& Pizzi (2002), o efeito do tratamento de termorretificação nas propriedades da madeira depende das variáveis do processo (temperatura, tempo, taxa de aquecimento, etc) e do material, no caso, da espécie, em razão da sua composição química e dos caracteres anatômicos específicos, como dimensões da amostra, teor de umidade da madeira, etc.

Neste contexto, o objetivo deste estudo foi avaliar o efeito de tratamentos termorretificadores nas propriedades físicas (densidade e teor de umidade) da madeira de teca (Tectona grandis L. f.).

\section{MATERIAL E MÉTODOS}

\subsection{Material e tratamento de termorretificação}

Árvores de teca (Tectona grandis L. f.), com 12 anos de idade, foram obtidas de um plantio localizado na área experimental do Instituto Federal de Educação, Ciência e Tecnologia do Mato Grosso, localizado em Cáceres, Mato Grosso. Foram selecionadas nove árvores provenientes de três diferentes espaçamentos: $4 \times 2 \mathrm{~m}, 5 \times 2 \mathrm{~m} \mathrm{e}$ $6 \times 2 \mathrm{~m}$, sendo consideradas três árvores para cada espaçamento.

As toras foram processadas e amostras de $150 \times$ $75 \times 20 \mathrm{~mm}$ (comprimento $\times$ largura $\times$ espessura) foram preparadas e divididas em dois grupos: (1) amostras com predominância de madeira de cerne ( $\geq 75 \%$ de madeira de cerne em relação ao alburno) e (2) amostras com 100\% de madeira de alburno. O material foi seco ao ar livre e, em seguida, acondicionado em câmara climática a $20^{\circ} \mathrm{C}$ e $65 \%$ de 
umidade relativa (UR), até atingir o teor de umidade de equilíbrio.

A termorretificação foi realizada em um forno mufla elétrico laboratorial, da marca Linn Elektro Therm, com dimensões de $600 \times 600 \times$ $700 \mathrm{~mm}$, equipado com um sistema de controle de temperatura e tempo. O tratamento foi realizado em quatro etapas, sendo selecionadas duas temperaturas finais de termorretificação: 180 e $200^{\circ} \mathrm{C}$. As quatro etapas do tratamento foram: (1) aquecimento do material até $100^{\circ} \mathrm{C}$ - período correspondente a 2 horas; (2) aumento da temperatura de $100^{\circ} \mathrm{C}$ até a temperatura final de termorretificação $\left(180\right.$ e $\left.200^{\circ} \mathrm{C}\right)$ - período correspondente a 30 minutos; (3) tempo de tratamento na temperatura de termorretificação 2 horas e 30 minutos, e (4) resfriamento do material durante aproximadamente 1 hora. A Figura 1 apresenta o ciclo total de tratamento, correspondente a 6 horas. O teor de umidade inicial das amostras foi de aproximadamente $8-10 \%$. Para cada batelada de tratamento, foram tratadas 15 amostras de madeira, as quais foram dispostas horizontalmente na parte inferior do forno mufla. Após o tratamento, as amostras foram acondicionadas em câmara climática a $20^{\circ} \mathrm{C}$ e $65 \%$ de UR, até atingir o teor de umidade de equilíbrio.

\subsection{Determinação das propriedades físicas}

As propriedades físicas avaliadas foram a densidade aparente e o teor de umidade de equilíbrio da madeira, antes e após os tratamentos termorretificadores. A densidade aparente foi determinada de acordo com a norma ASTM D 2395 (ASTM, 1999).

Os teores de umidade da madeira foram determinados através do medidor de umidade M51 da MARRARI Automação (Curitiba, Paraná), antes e após os tratamentos termorretificadores. Para tal, foi realizada uma varreadura na superfície de cada amostra e então utilizada a média dos valores de umidade obtidos.

As densidades obtidas antes e após os tratamentos termorretificadores - DENS e DENStermo, respectivamente - assim como os teores de umidade obtidos antes e após os tratamentos termorretificadores - TUE e TUE-termo, respectivamente - foram mensurados a partir das amostras climatizadas a $20^{\circ} \mathrm{C}$ e $65 \%$ de UR.

\subsection{Análises estatísticas}

Os dados obtidos para as propriedades físicas (DENS, DENS-termo, TUE e TUE-termo) foram submetidos a uma análise de variância (ANOVA)

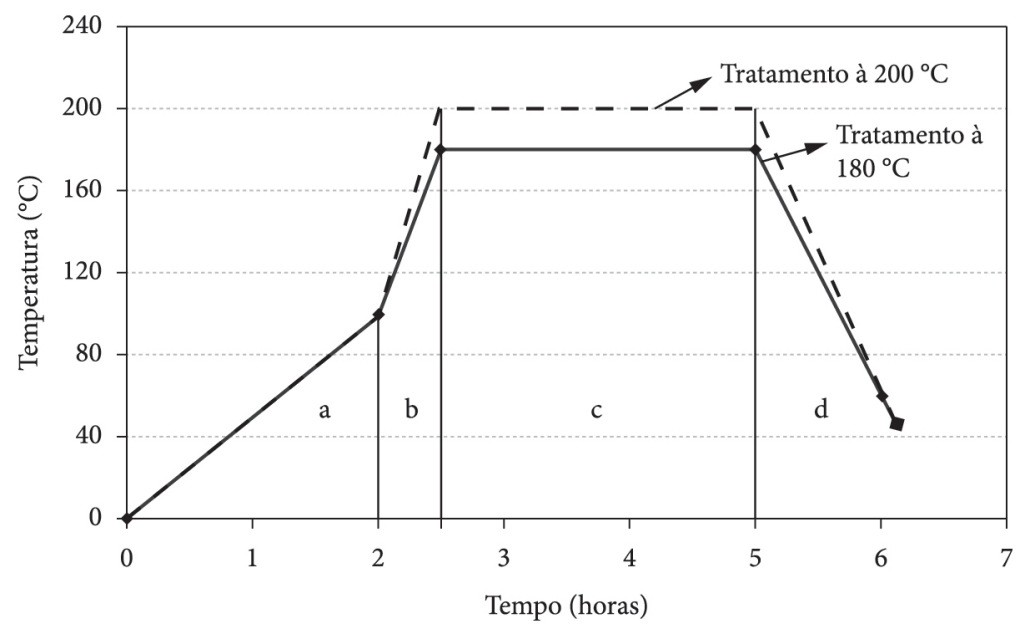

Figura 1. Programa de temperatura vs. tempo dos tratamentos termorretificadores aplicados à madeira de teca. a: aquecimento até $100^{\circ} \mathrm{C}$. b: aumento da temperatura de $100^{\circ} \mathrm{C}$ até a temperatura final de termorretificação ( 180 ou $\left.200^{\circ} \mathrm{C}\right)$. c: tratamento de termorretificação. d: resfriamento.

Figure 1. Program of temperature vs. time for heat treatments applied to teak wood. a: heating up to $100^{\circ} \mathrm{C}$. b: increase of temperature from $100^{\circ} \mathrm{C}$ to final temperature $\left(180\right.$ or $\left.200^{\circ} \mathrm{C}\right)$. c: heat treatment. d: cooling. 
com o auxílio do programa STATISTIC 6.0, a fim de determinar o efeito das variáveis independentes. Quando o valor $F$ foi significativo $(\alpha=0,05)$, aplicou-se o teste de Tukey a 95\% de significância, a fim de determinar as diferenças entre as médias das variáveis estudadas.

As distribuiçõoes das variáveis foram analisadas pelo teste Kolmogorov-Smirnov, em nível de 5\% de significância, para verificar a normalidade das suas distribuições.

As variáveis DENS e DENS-termo não apresentaram distribuição normal; portanto, aplicou-se a transformação logarítmica dos dados $\left(\log _{10}\right.$ DENS e $\log _{10}$ DENS-termo).

Neste estudo, foram consideradas as seguintes variáveis independentes: espaçamento entre árvores ( 3 níveis: $4 \times 2 \mathrm{~m}, 5 \times 2 \mathrm{~m} \mathrm{e} 6 \times 2 \mathrm{~m}$ ); grupo de madeira ( 2 níveis: cerne e alburno), e temperatura de termorretificação (2 níveis: 180 e $200^{\circ} \mathrm{C}$ ). Este delineamento experimental resultou em 12 tratamentos com cinco repetições, resultando em um total de 60 observações.

\section{RESULTADOS E DISCUSSÃO}

\subsection{Densidade da madeira antes e após a termorretificação}

Os resultados obtidos para a análise de variância das propriedades estudadas são apresentados na Tabela 1. Os valores médios obtidos para a densidade aparente da madeira de teca antes e após a termorretificação são apresentados na Tabela 2 .
As análises estatísticas indicaram uma interação significativa entre espaçamento e grupo para a densidade antes do tratamento (DENS) (Tabela 1 e Figura 2). Ao comparar os três espaçamentos, observa-se que a densidade da madeira de cerne não apresentou diferenças significativas. Entretanto, para o alburno, observa-se uma diferença significativa entre a densidade da madeira do espaçamento mais adensado $(4 \times 2 \mathrm{~m})$ e a densidade dos demais espaçamentos, apresentando o maior valor médio de densidade, 0,663 g.cm ${ }^{-3}$ (Tabela 2).

Ao comparar a densidade da madeira do cerne com o alburno, observa-se que esta apresenta um comportamento diferenciado somente no espaçamento mais adensado $(4 \times 2 \mathrm{~m})$, com valores médios de 0,594 e 0,663 g.cm ${ }^{-3}$, para cerne e alburno, respectivamente. Portanto, a madeira de alburno

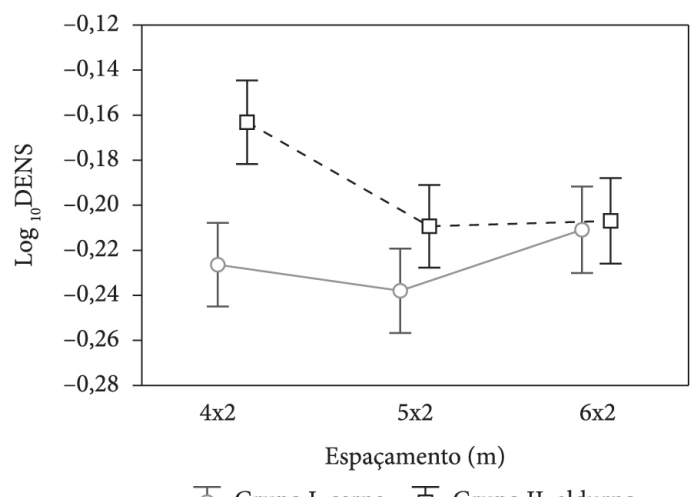

모 Grupo I: cerne 颔 Grupo II: aldurno

Figura 2. Interação entre espaçamento e grupo para a densidade da madeira de teca antes da termorretificação (DENS).

Figure 2. Interaction between spacing and group for density of teak wood before heat treatment.

Tabela 1. Análise de variância (valor de F) das propriedades físicas da madeira de teca antes e após a termorretificação. Table 1. Analysis of variance (F value) for physical properties of teak wood before and after heat treatment.

\begin{tabular}{lcccc}
\multicolumn{1}{c}{ Fonte de variação } & $\log _{10}$ DENS & $\begin{array}{c}\log _{10} \text { DENS- } \\
\text { termo }\end{array}$ & TUE & TUE-termo \\
\hline Espaçamento & $4,62^{*}$ & $3,98^{*}$ & $3,59^{*}$ & $0,46^{\text {ns }}$ \\
Grupo & $16,84^{* *}$ & $24,79^{* *}$ & $1,92^{\text {ns }}$ & $72,39^{* *}$ \\
Temperatura & - & $0,009^{\text {ns }}$ & - & $25,75^{* *}$ \\
Espaçamento $\times$ Grupo & $4,72^{*}$ & $4,69^{*}$ & $10,69^{* *}$ & $0,22^{\text {ns }}$ \\
Espaçamento $\times$ Temperatura & - & $0,098^{\text {ns }}$ & - & $1,59^{\text {ns }}$ \\
Grupo $\times$ Temperatura & - & $0,911^{\text {ns }}$ & - & $1,54^{\text {ns }}$ \\
Espaçamento $\times$ Grupo $\times$ Temperatura & - & $2,99^{\text {ns }}$ & - & $8,75^{* *}$ \\
\hline
\end{tabular}

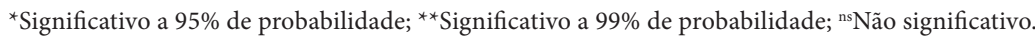


Tabela 2. Análises estatísticas descritivas (média e desvio padrão) das propriedades físicas (densidade aparente e teor de umidade) da madeira de teca antes e após a termorretificação.

Table 2. Descriptive statistic analysis (means and standard deviation) of the physical properties (density and moisture content) of teak wood before and after heat treatment.

\begin{tabular}{|c|c|c|c|c|c|c|c|c|}
\hline $\begin{array}{c}\text { Espaça- } \\
\text { mento } \\
(\mathrm{m})\end{array}$ & Grupo & $\begin{array}{l}\text { Tempera- } \\
\text { tura }\left({ }^{\circ} \mathrm{C}\right)\end{array}$ & $\begin{array}{c}\text { DENS } \\
\left({\left.\mathrm{g} . \mathrm{cm}^{-3}\right)}^{-3}\right.\end{array}$ & $\begin{array}{l}\log _{10} \\
\text { DENS }\end{array}$ & $\begin{array}{c}\text { DENS- } \\
\text { termo } \\
\left(\mathrm{g} \cdot \mathrm{cm}^{-3}\right)\end{array}$ & $\begin{array}{c}\log _{10} \\
\text { DENS- } \\
\text { termo }\end{array}$ & TUE (\%) & $\begin{array}{c}\text { TUE-ter- } \\
\text { mo (\%) }\end{array}$ \\
\hline \multirow{6}{*}{$4 \times 2$} & \multirow{3}{*}{ Cerne } & Controle & $0,594(0,018)$ & $-0,226 \mathrm{a}$ & - & - & 9,0 a $(0,34)$ & - \\
\hline & & 180 & - & - & $0,561(0,012)$ & $-0,251 \mathrm{a}$ & - & $4,9 \mathrm{~cd}(0,35)$ \\
\hline & & 200 & - & - & $0,528(0,019)$ & $-0,277 \mathrm{a}$ & 3,3 a $(0,41)$ & \\
\hline & \multirow{3}{*}{ Alburno } & Controle & $0,663(0,028)$ & $-0,163 b$ & - & - & $9,2 \mathrm{ab}(0,81)$ & - \\
\hline & & 180 & - & - & $0,644(0,036)$ & $-0,268 \mathrm{ab}$ & - & $5,1 \mathrm{~d}(0,34)$ \\
\hline & & 200 & - & - & $0,682(0,015)$ & $-0,234 \mathrm{~b}$ & - & $5,2 \mathrm{~d}(0,52)$ \\
\hline \multirow{6}{*}{$5 \times 2$} & \multirow{3}{*}{ Cerne } & Controle & $0,575(0,023)$ & $-0,238 \mathrm{a}$ & - & - & $10,0 \mathrm{~b}(0,87)$ & - \\
\hline & & 180 & - & - & $0,539(0,013)$ & $-0,268 \mathrm{a}$ & - & $4,1 \mathrm{c}(0,15)$ \\
\hline & & 200 & - & - & $0,520(0,034)$ & $-0,234 \mathrm{a}$ & $4,0 \mathrm{abc}(0,46)$ & \\
\hline & \multirow{3}{*}{ Alburno } & Controle & $0,605(0,034)$ & $-0,208 \mathrm{a}$ & - & - & 8,7 a $(0,38)$ & - \\
\hline & & 180 & - & - & $0,579(0,042)$ & $-0,240 \mathrm{ab}$ & - & $4,8 \mathrm{~d}(0,17)$ \\
\hline & & 200 & - & - & $0,593(0,044)$ & $-0,234 \mathrm{ab}$ & - & 4,3 bcd $(0,77)$ \\
\hline \multirow{6}{*}{$6 \times 2$} & \multirow{3}{*}{ Cerne } & Controle & $0,616(0,017)$ & $-0210 a$ & - & - & $\begin{array}{l}9,4 \mathrm{abc} \\
(0,38)-\end{array}$ & \\
\hline & & 180 & - & - & $0,555(0,012)$ & $-0,255 \mathrm{a}$ & - & 4,5 bcd $(0,61)$ \\
\hline & & 200 & - & - & $0,560(0,018)$ & $-0,245 \mathrm{a}$ & - & $4,1 \mathrm{bc}(0,34)$ \\
\hline & \multirow{3}{*}{ Alburno } & Controle & $0,624(0,050)$ & $-0,207 a$ & - & - & 9,8 bc $(0,32)$ & - \\
\hline & & 180 & - & - & $0,536(0,028)$ & $-0,232 \mathrm{ab}$ & - & $5,4 \mathrm{~d}(0,40)$ \\
\hline & & 200 & - & - & $0,560(0,055)$ & $-0,249 a$ & - & $4,6 \mathrm{bc}(0,52)$ \\
\hline
\end{tabular}

DENS, DENS-termo: densidade aparente da madeira antes e após a termorretificação, respectivamente; TUE, TUE-termo: teor de umidade da madeira antes e após a termorretificação, respectivamente. Os valores entre parênteses referem-se ao desvio padrão. Médias com a mesma letra não são estatisticamente diferentes entre si segundo o teste de Tukey. As comparações entre as médias são feitas na mesma coluna.

é mais densa do que a madeira do cerne nesse espaçamento.

De acordo com a literatura, a madeira de teca adulta apresenta um valor médio de densidade próximo a 0,650 g.cm ${ }^{-3}$ (Lincoln, 1986). Os valores médios de densidade encontrados neste estudo são inferiores, exceto para a madeira de alburno do espaçamento de $4 \times 2 \mathrm{~m}$. Esses resultados podem ser explicados pela idade das árvores de teca utilizadas neste estudo, as quais são consideradas jovens com 12 anos de idade, sendo, provavelmente, composta apenas por madeira juvenil.

Souza (2010) estudou a variação do comprimento de fibras, no sentido medula-casca, para caracterizar a transição entre os lenhos juvenil e adulto na madeira de teca de 12 anos de idade provenientes do mesmo plantio e observou que o comprimento das fibras não se estabilizou, deduzindo-se que a madeira ainda não possuía lenho adulto. Entretanto, o autor observou diferenças significativas para o comprimento e a espessura de parede das fibras em função da posição radial, ou seja, ocorreu um aumento da espessura de parede na direção medula-casca. Os valores médios obtidos para o comprimento das fibras próximo à medula e à casca foram de 814,0 e 1177,4 $\mu \mathrm{m}$, respectivamente, enquanto que os valores médios obtidos para espessura de parede foram de 4,0 e $5,3 \mu \mathrm{m}$, respectivamente.

Souza (2010) observou ainda que o comprimento das fibras e a espessura de parede no espaçamento 6 $\times 2 \mathrm{~m}$ foram diferentes dos espaçamentos $4 \times 2 \mathrm{~m} \mathrm{e}$ $5 \times 2 \mathrm{~m}$, sendo que, nos plantios mais adensados, o volume de madeira produzido por unidade de área era menor, com fibras de menor comprimento e maior espessura de parede.

O efeito do espaçamento nos caracteres anatômicos da madeira de teca, apresentados por Souza (2010), pode explicar a razão pela qual a madeira de alburno, no espaçamento mais adensado $(4 \times 2 \mathrm{~m})$, apresentou um valor maior para a 


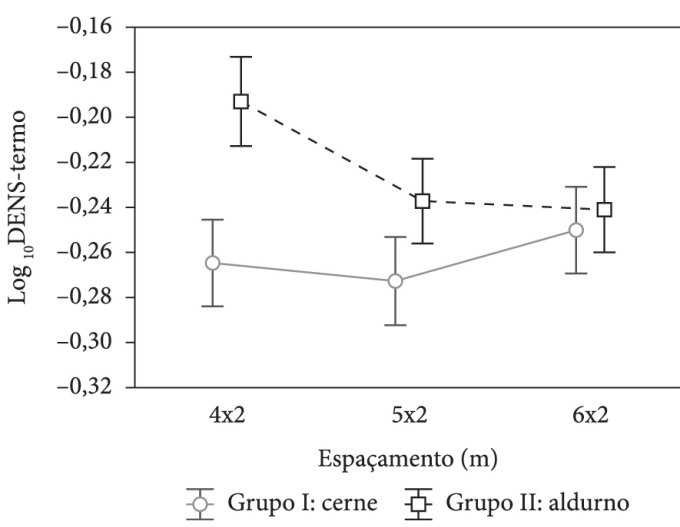

Figura 3. Interação entre espaçamento e grupo para a densidade da madeira de Tectona grandis após a termorretificação (DENS-termo).

Figure 3. Interation between spacing and group for density of teak wood after heat treatment (DENStermo).

densidade. A diferença entre cerne (próximo à porção mais interna do tronco) e alburno (próximo à casca) para o espaçamento $4 \times 2 \mathrm{~m}$ corrobora com os resultados obtidos por Garcia et al. (2009), que observaram um aumento da densidade no sentido radial (direção medula-casca).

A densidade da madeira de teca termorretificada (DENS-termo) apresentou um decréscimo em ambos os grupos (cerne e alburno) quando comparada à densidade antes do tratamento (DENS) (Tabela 2); porém, DENS-termo não foi afetada significativamente pela temperatura do tratamento, tal como verificado na Tabela 1.

As análises estatísticas mostraram uma interação significativa entre espaçamento e grupo para a densidade após o tratamento (DENS-termo) (Tabela 2 e Figura 3).

Ao comparar os grupos, verifica-se que a madeira de cerne apresentou um decréscimo médio de 8,67\% na densidade após a termorretificação, enquanto que a madeira de alburno apresentou um decréscimo médio de $6 \%$. Assim, o cerne apresentou uma redução maior de densidade em relação ao alburno após a termorretificação. Metsä-Kortelainen et al. (2005) encontraram uma perda semelhante para a densidade do cerne e do alburno de Pinus sylvestris, quando tratados a $210^{\circ} \mathrm{C}$, com perdas de densidade de $6,9 \%$ e $6,7 \%$, respectivamente. A maior redução da densidade na madeira do cerne pode ser explicada

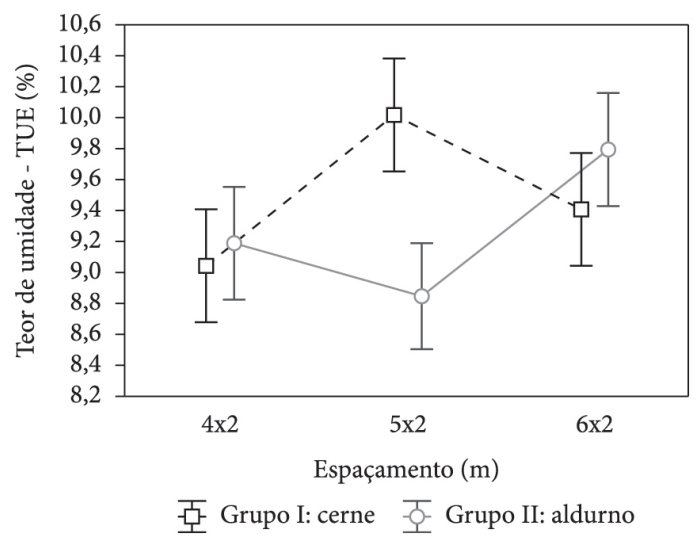

Figura 4. Interação entre espaçamento e grupo para o teor de umidade da madeira de teca antes da termorretificação (TUE).

Figure 4. Interaction between spacing and group for moisture content of teak wood before heat treatment (TUE).

pelo maior teor de extrativos, os quais se volatilizam durante o tratamento, causando assim maiores perdas de peso.

\subsection{Teor de umidade da madeira antes e após a termorretificação}

Para o teor de umidade da madeira original (TUE), as análises estatísticas indicaram diferenças significativas entre os espaçamentos e uma interação significativa entre espaçamento e grupo (Tabela 1). Esta interação pode ser observada na Figura 4.

Comparando-se os teores de umidade encontrados dentro dos espaçamentos, observase uma diferença significativa somente entre as madeiras de cerne e alburno do espaçamento de 5 $\times 2 \mathrm{~m}$, sendo que o teor de umidade apresentou-se maior na madeira de cerne. Ao se comparar TUE do cerne entre os espaçamentos, observam-se diferenças significativas somente entre os espaçamentos de $4 \times 2 \mathrm{~m}$ e $5 \times 2 \mathrm{~m}$; já para a madeira de alburno, encontraram-se diferenças significativas entre os espaçamentos $5 \times 2 \mathrm{me} 6 \times 2 \mathrm{~m}$ (Tabela 2).

Após a termorretificação, o teor de umidade (TUE-termo) sofreu um decréscimo médio de 52,1\%. As análises estatísticas indicaram uma interação tripla (espaçamento $\times$ grupo $\times$ temperatura) para TUE-termo (Tabela 1; Figura 5). Rousset et al. (2004) obtiveram resultados similares para a madeira de 


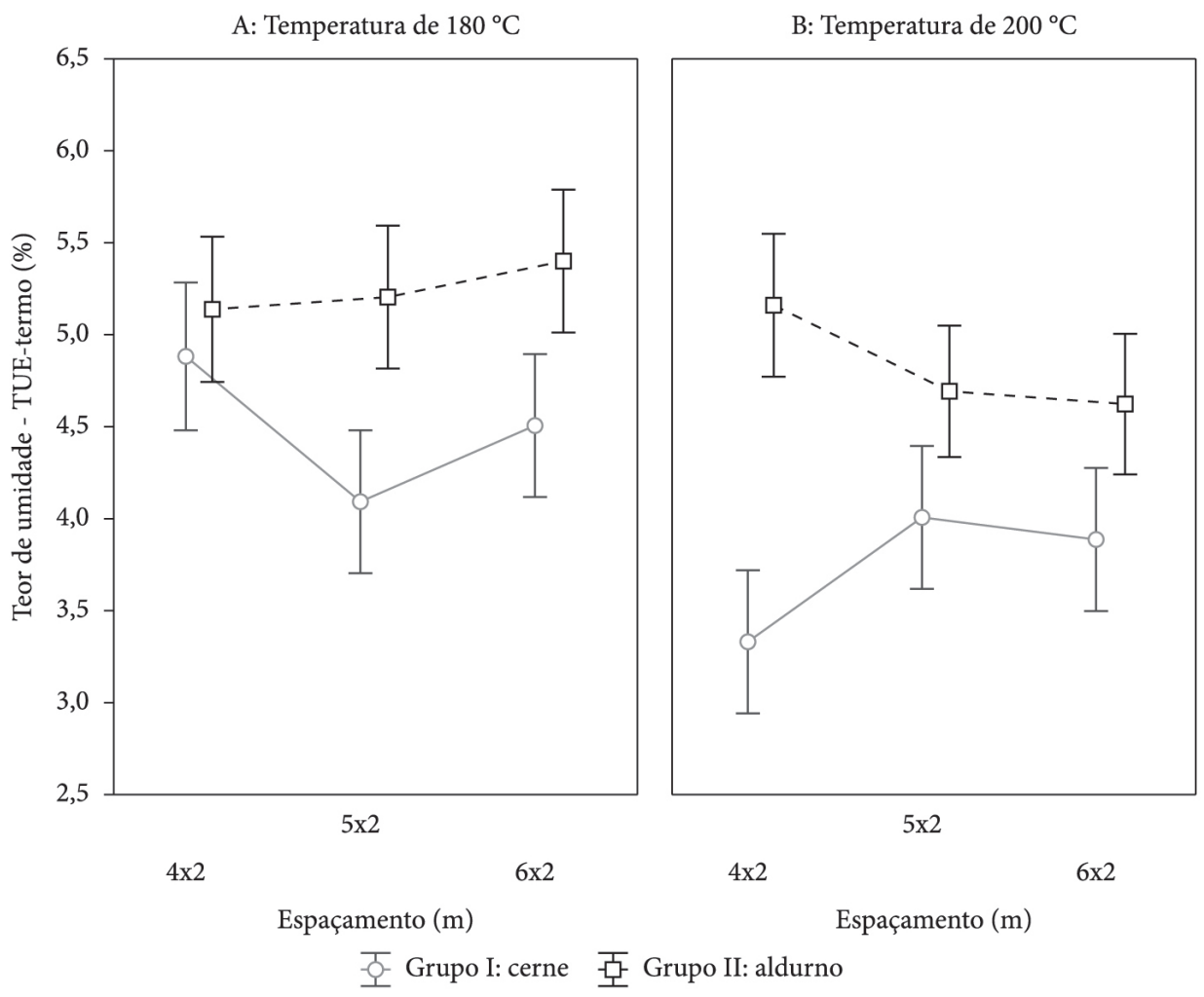

Figura 5. Interação tripla (espaçamento $\times$ grupo $\times$ temperatura) para o teor de umidade da madeira de teca após a termorretificação (TUE-termo).

Figure 5. Triple interaction (spacing $\mathrm{x}$ group $\mathrm{x}$ temperature) for moisture content of teak wood after heat treatment (TUE-termo).

Populus robusta tratada a $200^{\circ} \mathrm{C}$ durante 1 hora, a qual apresentou reduções significativas entre 40 e $60 \%$.

A madeira de cerne sofreu um decréscimo médio de 53,5\% no teor de umidade após a termorretificação, sendo que a temperatura mostrou um efeito somente no espaçamento de $4 \times 2 \mathrm{~m}$, no qual a temperatura de $180^{\circ} \mathrm{C}$ promoveu um decréscimo menor no TUE-termo do que a temperatura de $200^{\circ} \mathrm{C}$ (Tabela 2). O teor de umidade da madeira tratada a $180^{\circ} \mathrm{C}$ apresentou um decréscimo de $46 \%$ quando comparado ao valor inicial, enquanto que, a $200^{\circ} \mathrm{C}$, o teor de umidade sofreu um decréscimo de $54 \%$, sendo também o menor valor obtido para o teor de umidade (Tabela 2).

Já a madeira de alburno sofreu um decréscimo médio de $47 \%$ no teor de umidade, sendo o efeito da temperatura significativo somente para o espaçamento de $6 \times 2 \mathrm{~m}$. Neste caso, o teor de umidade da madeira tratada a $180^{\circ} \mathrm{C}$ apresentou um decréscimo de $45 \%$, enquanto que, a $200^{\circ} \mathrm{C}$, o teor de umidade da madeira foi reduzido em $54 \%$. Esses resultados são similares àqueles relatados por Jämsä \& Viitameni (2001), os quais afirmam que as madeiras tratadas termicamente apresentam reduções de até $50 \%$ no teor de umidade de equilíbrio higroscópico, devido à diminuição dos sítios de adsorção (região amorfa) e à degradação parcial das hemiceluloses, as quais são os polímeros mais higroscópicos da parede celular.

Os resultados encontrados no presente estudo também são coerentes com a afirmação feita por Vernois (2001) de que as madeiras tratadas termicamente, em torno de $200^{\circ} \mathrm{C}$, além de apresentarem higroscopicidade menor do que as não tratadas, se estabilizaram em torno de $4-5 \%$ de umidade, em vez de $10-12 \%$. 


\section{CONCLUSÕES}

O espaçamento afetou as propriedades físicas da madeira de teca após a termorretificação.

A madeira de cerne apresentou um decréscimo médio de $8,67 \%$ na densidade após a termorretificação, enquanto que a madeira de alburno apresentou um decréscimo médio de $6 \%$. Dessa forma, o cerne apresentou uma redução maior de densidade em relação ao alburno, após a termorretificação.

As madeiras de cerne e alburno apresentaram uma redução média de $52 \%$ no teor de umidade de equilíbrio, após a termorretificação.

A temperatura mostrou efeito para a madeira de cerne no espaçamento $4 \times 2 \mathrm{~m}$. O aumento da temperatura promoveu decréscimos significativos e respectivos de $46 \%$ e $54 \%$.

A temperatura mostrou efeito para a madeira de alburno no espaçamento $6 \times 2$. O aumento da temperatura promoveu decréscimos significativos e respectivos de $45 \%$ e $54 \%$.

Portanto, após a termorretificação, as madeiras de cerne e alburno sofreram decréscimo no teor de umidade de equilíbrio, sendo que o aumento da temperatura tornou esse efeito mais pronunciado.

\section{STATUS DA SUBMISSÃO}

Recebido: 23 abr., 2013

Aceito: 17 jul., 2014

Publicado: 21 nov., 2014

\section{AUTOR(ES) PARA CORRESPONDÊNCIA}

\section{Juliana Oliveira Lopes}

Programa de Pós-Graduação em Ciências

Ambientais e Florestais, Universidade Federal Rural do Rio de Janeiro - UFRRJ, CEP 23890000, Seropédica, RJ, Brasil

e-mail: july.rural@gmail.com

\section{REFERÊNCIAS}

American Society for Testing and Materials - ASTM. D 2395-93: standard test methods for specific gravity of wood and wood-based materials. Philadelphia; 1999.
Esteves B, Domingos I, Pereira H. Improvement of technological quality of eucalypt wood by heat treatment in air at $170-200{ }^{\circ} \mathrm{C}$. Forest Products Journal 2007; 57(1-2): 47-52.

Garcia RA, Carvalho AM, Latorraca JVF, Matos JLM, Santos WA, Silva RFM. Nondestructive evaluation of heat-treated Eucalyptus grandis Hill ex Maiden wood using stress wave method. Wood Science and Technology 2012; 46(1-3): 41-52. http://dx.doi.org/10.1007/s00226010-0387-6

Garcia R, Lima IL, Longui EL, Florsheim SMB. Influência do espaçamento e da posição radial na variação de elementos anatômicos da madeira de Tectona grandis Linn. São Paulo: Instituto Florestal; 2009. p. 5156. IF Série Registros n. 40.

Hakkou M, Pétrissans M, El Bakali I, Gérardin P, Zoulalian A. Wettability changes and mass loss during heat treatment of wood. Holzforschung 2005; 59(1): 3537. http://dx.doi.org/10.1515/HF.2005.006

Jämsä S, Viitaniemi P. Heat treatment of wood: better durability with out chemicals. In: Rapp AO. Review on heat treatments of wood. In: Special Seminar: Environmental Optimization of Wood Protection; 2001; Antibes, France. Antibes: COST Action E22; 2001. p. 21-26.

Kamdem DP, Pizzi A, Jermannaud A. Durability of heattreated wood. Holz als Roh-und Werkstoff 2002; 60(1): 1-6. http://dx.doi.org/10.1007/s00107-001-0261-1

Lincoln WA. World woods in colour. Hertford: Stobart Davies; 1986.

Lopes JO. Uniformidade e estabilidade da cor da madeira termorretificada de Tectona grandis L. f. [dissertação]. Seropédica: Universidade Federal Rural do Rio de Janeiro; 2012.

Metsä-Kortelainen S, Anitikainen T, Viitaniemi P. The water absorption of sapwood and heartwood of Scots pines and Norway spruce heat-treated at $170{ }^{\circ} \mathrm{C}, 190^{\circ} \mathrm{C}$, $210^{\circ} \mathrm{C}$ and $230^{\circ} \mathrm{C}$. Holz als Roh- und Werkstoff 2005; 64(3): 192-197. http://dx.doi.org/10.1007/s00107-0050063-y

Militz, R. Heat treatment technologies in Europe: Scientific Background and technological state-of-art. In: Conference on enhancing the durability of lumber and Engineered Wood Products; 2002; Kissimmee, Orlando. Kissimmee: Forest Products Society; 2002.

Möttönen K, AlvilaL, Pakkanen T.CIELab Measurements to determine the role of felling season, log storage and kiln drying on coloration of silver birch wood. Scandinavian Journal Forest Research 2002; 17(2): 179191. http://dx.doi.org/10.1080/028275802753626827 
Oltean L, Teischinger A, Hansmann C. Influence of temperature on cracking and mechanical properties of wood during wood drying: a review. BioResources 2007; 2(4): 789-811.

Rapp AO. Review on heat treatments of wood. In: Special Seminar: Environmental Optimization of Wood Protection; 2001; Antibes, France. Antibes, COST Action E22; 2001.68 p.

Rousset P, Perré P, Girard P. Modification of mass transfer properties in poplar wood ( $P$. robusta) by thermal treatment at high temperature. Holz als Rohund Werkstoff 2004; 62(2): 113-119. http://dx.doi. org/10.1007/s00107-003-0459-5

Souza MT. Influência do espaçamento e da posição radial nas dimensões das fibras da madeira de Tectona grandis L. f. [monografia]. Seropédica: Universidade Federal Rural do Rio de Janeiro; 2010.
Syrjänen T. Production and classification of heat treated wood in Finland. In: Rapp AO. Review on heat treatments of wood. In: Special Seminar: Environmental Optimization of Wood Protection; 2001; Antibes, France. Antibes, COST Action E22; 2001. p.11-20.

Vernois M. Heat treatment of wood in France: state of the art. In: Rapp AO. Review on Heat Treatments of Wood. In: Special Seminar: Environmental Optimization of Wood Protection; 2001; Antibes, France. Antibes, COST Action E22; 2001.68 p.

Viitaniemi P, Jamsa S. Modification of wood with heat treatment. Espoo: VTT; 1994. VTT Research Report Publications, 814.

Weiland JJ, Guyonnet R. Study of chemical modifications and fungi degradation of thermally modified wood using DRIFT spectroscopy. Holz als Roh-und Werkstoff 2003; 61(3): 216-220. 